\title{
Effect of the BTK inhibitor ibrutinib on macrophage- and $\gamma \delta$ T cell-mediated response against Mycobacterium tuberculosis
}

\author{
Ana Colado ${ }^{1}$, Melanie Genoula ${ }^{2,3,4}$, Céline Cougoule ${ }^{3,4,5}$, José L. Marín Franco ${ }^{2,3,4}$, María B. Almejún 1,6, Denise Risnik' , \\ Denise Kviatcovsky ${ }^{2,3,4}$, Enrique Podaza', Esteban E. Elías' ${ }^{1}$, Federico Fuentes ${ }^{7}$, Isabelle Maridonneau-Parini, ${ }^{3,4,5}$, \\ Fernando R. Bezares ${ }^{8}$, Horacio Fernandez Grecco ${ }^{9}$, María Cabrejo ${ }^{9}$, Carolina Jancic ${ }^{10,11}$, María del Carmen Sasiain ${ }^{2,3,4}$, \\ Mirta Giordano ${ }^{1,11}$, Romina Gamberale ${ }^{1,11}$, Luciana Balboa ${ }^{2,3,4}$ and Mercedes Borge $\mathbb{1}^{1,11}$
}

The Bruton's tyrosine kinase (BTK) inhibitor ibrutinib is approved by the Food and Drug Administration for its use as first-line treatment in chronic lymphocytic leukemia (CLL). Despite its efficacy, patients treated with ibrutinib rarely achieve complete responses and usually remain under treatment until progression. Considering the inherent high susceptibility of CLL patients to infections, a better understanding of ibrutinib effects on the immune system might help to estimate the risk of infectious complications on treated patients. Besides its effects on leukemic B cells, ibrutinib also affects functions on T cells, natural killer cells, and macrophages ${ }^{1-3}$. Macrophages are central players of the innate immune response against fungi, extracellular bacteria, and in particular against the intracellular bacteria Mycobacterium tuberculosis (Mtb). The World Health Organization (WHO) estimates that one-third of the world's population is infected with $M t b$, the causing agent of tuberculosis, a severe infection that kills near 1.3 million people per year and the leading cause of death from a single infectious agent (Global TB report, WHO, 2017). Notably, the incidence rate of tuberculosis is highly variable among different countries. In South American countries, such as Argentina and Brazil, where ibrutinib is being introduced, the rates of tuberculosis

\footnotetext{
Correspondence: Mercedes Borge (mercedesborge@hotmail.com)

'Laboratorio de Inmunología Oncológica, Instituto de Medicina Experimental (IMEX)-CONICET-Academia Nacional de Medicina (ANM), CABA, Argentina

${ }^{2}$ Laboratorio de Inmunología de Enfermedades Respiratorias, IMEX-CONICETANM, CABA, Argentina
}

Full list of author information is available at the end of the article. incidence are up to 14 times higher than in USA and other developed countries. Given the relevance of macrophages in $M t b$ immune response, we here evaluated the in vitro effects of ibrutinib on this cell compartment. Additionally, we studied its effects on $\gamma \delta \mathrm{T}$ cells, another innate immune component reported to be involved in $M t b$ response ${ }^{4}$. Strikingly, we found that ibrutinib affects macrophage's phenotype and the response of both macrophages and $\gamma \delta \mathrm{T}$ cells to $M t b$.

Macrophages were differentiated from human monocytes with macrophage colony-stimulating factor (MCSF), then pre-treated with ibrutinib for $30 \mathrm{~min}$, and afterward exposed to irradiated $M t b$ for $24 \mathrm{~h}$. We found that clinically relevant doses of ibrutinib $(0.03-0.3 \mu \mathrm{M})$ significantly reduced the release of tumor necrosis factor (TNF) $-\alpha$ (Fig. 1a and Supplementary Fig. 1a), while interleukin (IL)-10 and IL-8 secretion was only affected at $3 \mu \mathrm{M}$, which is a concentration higher than the one reported in the plasma of treated patients (Fig. 1b, c). Importantly, macrophage viability was not affected by ibrutinib (Supplementary Fig. 1b). Inhibition of TNF- $\alpha$ secretion was associated with a diminished phosphorylation of the p65 subunit of the transcription factor nuclear factor (NF)-kB (Fig. 1d), a key regulator of cytokine production in macrophages. Given the relevance of Toll-like receptor (TLR) 2 and TLR4 in $M t b$ recognition by macrophages, we evaluated the effect of ibrutinib on TNF- $\alpha$ secretion in response to lipopolysaccharide (LPS), a TLR4 ligand, and Pam3CSK4, a TLR2 ligand. Results in Fig. 1e show that ibrutinib impaired TNF- $\alpha$ secretion induced by 

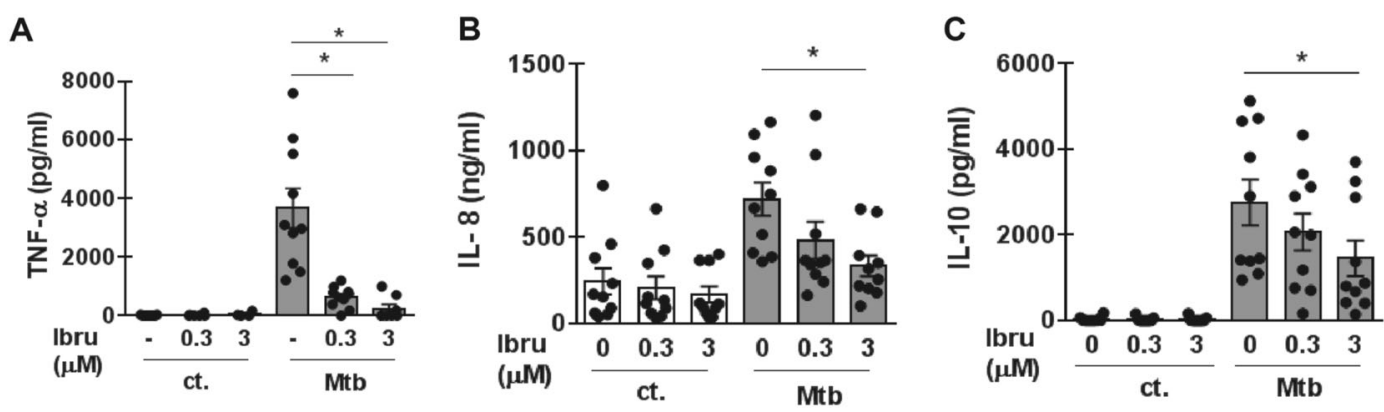

D
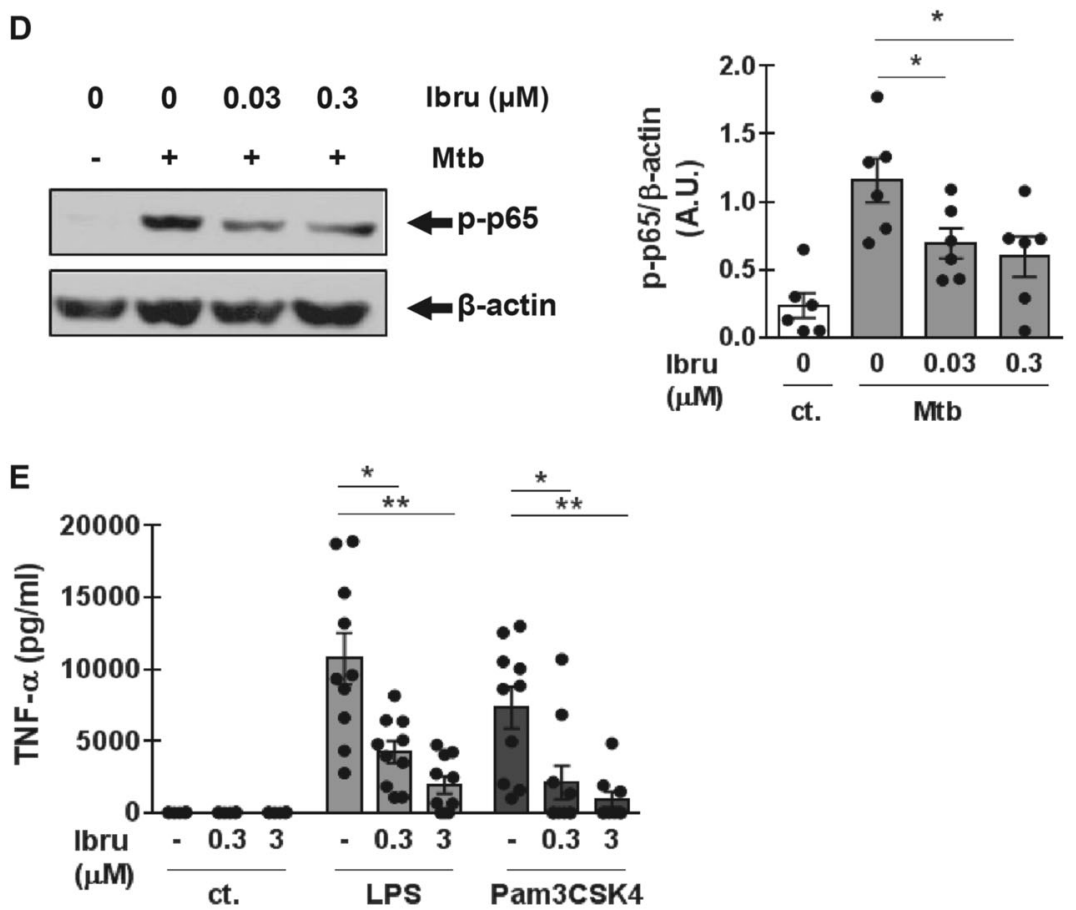

F CLL-derived macrophages
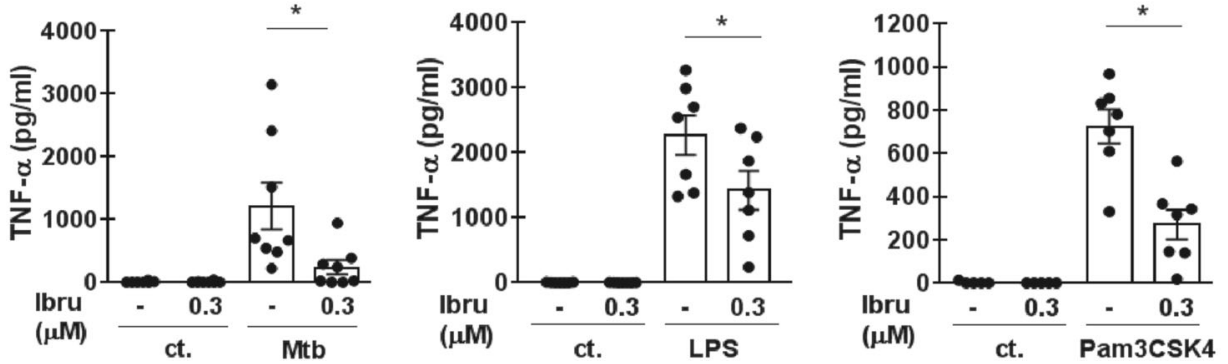

Fig. 1 lbrutinib impairs macrophage-mediated response against Mycobacterium tuberculosis. Macrophages were obtained by culturing monocytes from healthy donors (HD) or CLL patients for 5 days in RPMI 10\% FCS in the presence of M-CSF ( $50 \mathrm{ng} / \mathrm{ml})$. a-c HD-macrophages were stimulated with irradiated Mtb (MOI equivalent to 2) in the presence or absence of ibrutinib (Ibru) and after $24 \mathrm{~h} \mathrm{TNF-a,} \mathrm{IL-8,} \mathrm{and} \mathrm{IL-10} \mathrm{secretion} \mathrm{was}$ measured by ELISA in culture supernatants. Bars represent mean \pm SEM of cytokine concentration in control (ct.) cultures (white bars) or Mtbstimulated cultures (gray bars). $n=10,{ }^{*} p<0.05$, Kruskal-Wallis test, followed by Dunn's multiple comparisons test. $\mathbf{d}$ Phosphorylation of p65 ( $p$-p65) was evaluated by western blot in HD macrophages after 15 min of stimulation with Mtb in the presence or absence of ibrutinib. Bands on the immunoblots were quantified using the ImageJ software ( $\mathrm{NIH}$ Image). Results are shown as the mean \pm SEM of the ratio $p$-p65/ $\beta$-actin in arbitrary units (A.U.). $n=6,{ }^{*} p<0.05$, Friedman test, followed by Dunn's multiple comparisons test. e HD macrophages were stimulated with LPS (100 ng/ml) or Pam3CSK4 $(100 \mathrm{ng} / \mathrm{ml})$ in the presence or absence of ibrutinib for $24 \mathrm{~h}$ and TNF-a production was measured in culture supernatants by ELISA. $n=$ $10,{ }^{*} p<0.05$, Kruskal-Wallis test, followed by Dunn's multiple comparisons test. f CLL macrophages were stimulated with irradiated Mtb (MOI equivalent to 2), LPS $(100 \mathrm{ng} / \mathrm{ml})$, or Pam3CSK4 $(100 \mathrm{ng} / \mathrm{ml})$ in the presence or absence of ibrutinib for $24 \mathrm{~h}$ and TNF-a production was measured by ELISA in culture supernatants. $n=7,{ }^{*} p<0.05$, Kruskal-Wallis test, followed by Dunn's multiple comparisons test 
these ligands. Of note, the inhibition in response to TLR2, but not to TLR4, stimulation was observed even at low concentrations of ibrutinib ( $30 \mathrm{nM})$ (Supplementary Fig. 1c, d), suggesting a differential involvement of BTK, or other ibrutinib targets, in TLR2 and TLR4 signaling pathways. Inhibition of TNF- $\alpha$ secretion in response to $M t b$, Pam3CSK4, and LPS was also observed in ibrutinibtreated monocyte-derived macrophages from CLL patients (Fig. 1f). Clinical characteristics of CLL patients included in this study are presented in Supplementary Table 1.

Then, given that macrophages with different polarization profiles have different abilities to promote an efficient immune response against $M t b$, the M1 pro-inflammatory profile being more effective than the M2 antiinflammatory profile ${ }^{5}$, we aimed to evaluate the effect of ibrutinib on macrophage polarization in interferon (IFN)$\gamma$-induced M1, IL4-induced M2, and IL-10-induced M2 profiles. M1 and M2 polarization was confirmed by analyzing the expression of CD16, CD14, CD163, CD206, CD86, and HLA-DR by flow cytometry (Supplementary Fig. 2). We found that ibrutinib impaired M1 polarization as shown by the upregulation of M2-associated markers CD16, CD14, CD163, and CD206 and by downregulation of the M1-associated markers CD86 and HLA-DR (Fig. $2 a, b)$. The expression of these markers was not modified by ibrutinib on IL-4- and IL-10-induced M2 macrophages (Supplementary Fig. 3). These results are in line with those recently published by Fiocari et al. showing that ibrutinib promotes an M2 phenotype in nurse-like cells from CLL patients ${ }^{2}$. Impairment in M1 polarization, induced upon ibrutinib exposure, was not associated with a decrease in signal transducer and activator of transcription factor (STAT) 1 phosphorylation (Supplementary Fig. 4). Interestingly, we found that ibrutinib enhanced macrophage three-dimensional migration in Matrigel (Fig. 2c), a feature of macrophage with an M2 profile $^{6}$. We also found that M1 macrophages polarized in the presence of ibrutinib showed a decreased secretion of TNF- $\alpha$ and an increased secretion of IL-10 compared to control M1 macrophages, resembling the cytokine profile associated with M2 macrophages (Fig. 2d). M1 and M2 macrophages also differ in their glucose metabolic pathways. During M1 polarization, macrophages activate the aerobic glycolytic pathway, increasing glucose uptake and lactate production, while M2 macrophages preferentially use the oxidative metabolism to obtain energy. Importantly, this switch towards aerobic glycolysis seems to be necessary for an effective differentiation into the M1 profile$^{7}$. We found that ibrutinib treatment of M1 macrophages reduced both glucose consumption and lactate production (Fig. 2e). The impairment in M1 polarization, and the reduction in glucose consumption and lactate production induced by ibrutinib were also confirmed in macrophages from CLL patients (Supplementary Fig. 5). We also evaluated whether ibrutinib affects the functionality of polarized macrophages and found that ibrutinib decreased TNF- $\alpha$ production in response to $M t b$ and increased migration in matrigel in M1 macrophages (Supplementary Fig. 6). Taken together, these results showed that ibrutinib affects M1 polarization of macrophage and their function, which could have detrimental consequences on the immune response to $M t b$ in patients treated with ibrutinib.

Then, since treatment of macrophages with ibrutinib during M1 polarization increased CD206 expression (Fig. 2a), a receptor involved in $M t b$ phagocytosis, we evaluated whether macrophage phagocytosis and/or the intracellular growth of $M t b$ was affected in this situation. As shown in Fig. 2f, M1 macrophages polarized in the presence of ibrutinib showed a slight increase in $M t b$ uptake, while the intracellular growth of the bacteria was not modified (Supplementary Fig. 7). On the other hand, when we compared the effect of ibrutinib on the intracellular growth of already infected M1 macrophages, we found that ibrutinib impaired their killing capacity as shown by the increase in the bacillary load (Fig. 2g, h).

$\mathrm{T}$ cells bearing the $\gamma \delta \mathrm{T}$ cell receptor also participate in the innate host defense against $M t b . \gamma \delta \mathrm{T}$ cells are found in $M t b$-induced lesions in humans, they release IFN- $\gamma$ in response to $M t b$, a cytokine associated with protective immunity, and are cytotoxic against $M t b$-infected macrophages resulting in killing of intracellular bacilli ${ }^{4}$. Therefore, we evaluated the effect of ibrutinib on human $\gamma \delta \mathrm{T}$ cells stimulated with $M t b$ and found a significant decrease in the expression of the activation marker CD69 and in the secretion of IFN- $\gamma$ (Fig. 2i, j), suggesting that $\gamma \delta$ $\mathrm{T}$ cell response to $M t b$ might also be compromised in ibrutinib-treated patients.

Results presented here show that ibrutinib affects macrophages and $\gamma \delta \mathrm{T}$ cells, which are important players in an effective immune response to $M t b$. In particular, we observed that ibrutinib, used at doses found in the plasma of treated patients $(0.03-0.3 \mu \mathrm{M})$, impairs immune mechanisms that contribute to the control of $M t b$ infection such as TNF- $\alpha$ secretion by macrophages, M1 polarization, $M t b$ intracellular growth in M1 macrophages, and IFN- $\gamma$ secretion by $\gamma \delta \mathrm{T}$ cells. Importantly, the ability of the host to limit $M t b$ at the site of infection depends on the formation and maintenance of an effective granuloma structure. In this context, TNF- $\alpha$ production by activated macrophages plays a key role in $M t b$ control by promoting granuloma formation. In fact, patients treated with TNF- $\alpha$-blocking agents have an increased risk of tuberculosis and the assessment of latent infection is recommended before starting such treatment. Our results showing that ibrutinib significantly decrease TNFa secretion in response to $M t b$ suggests that this 


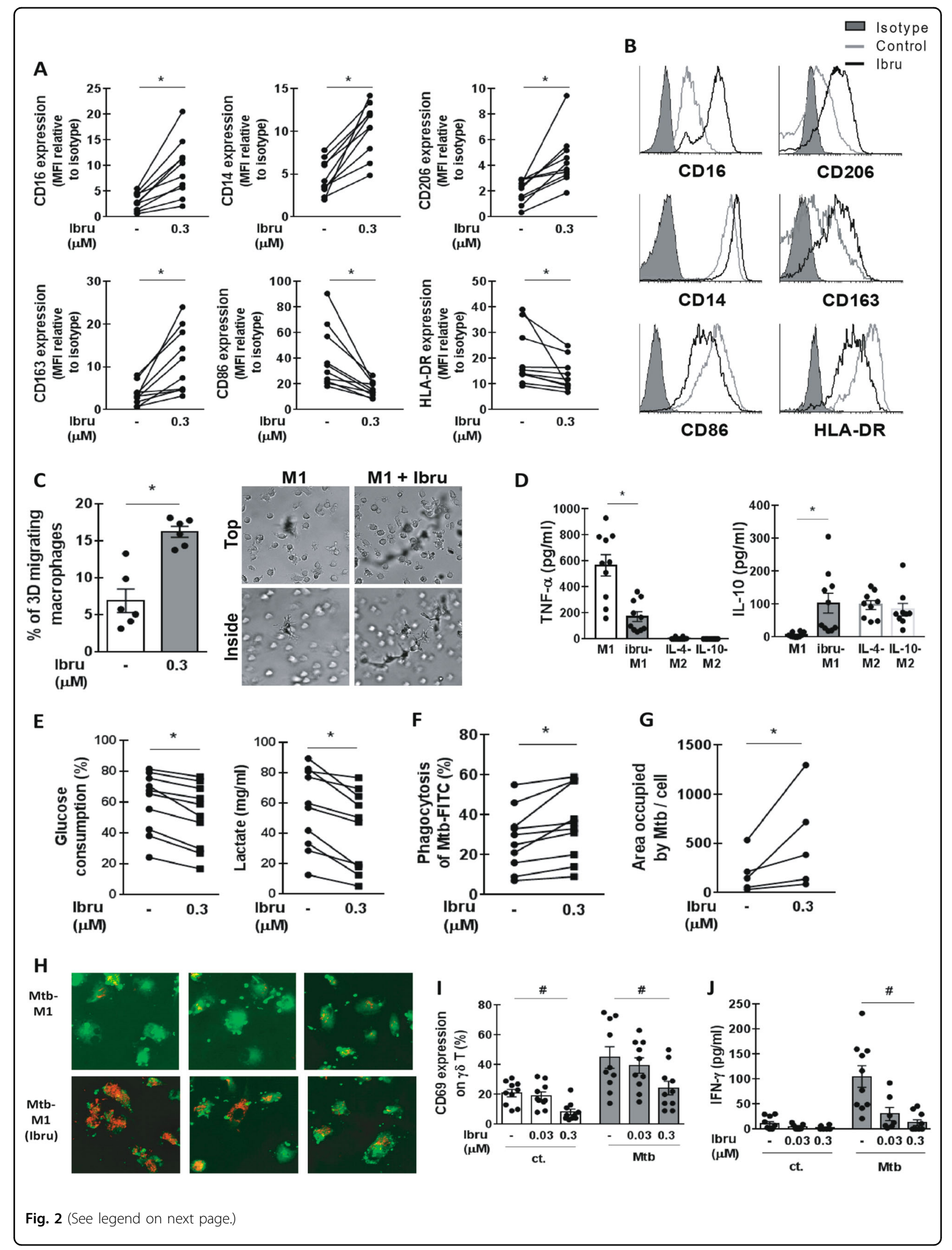




\begin{abstract}
(see figure on previous page)
Fig. 2 lbrutinib impairs $M 1$ polarization and affects macrophage and $\gamma \delta$ T cell response to Mycobacterium tuberculosis. To obtain the $M 1$ profile, monocytes from HD were cultured with GM-CSF $(50 \mathrm{ng} / \mathrm{ml})$ for 7 days and IFN- $\gamma(10 \mathrm{ng} / \mathrm{ml})$ was added for the last 2 days of culture. Ibrutinib $(0.3 \mu \mathrm{M})$ was added to the culture $30 \mathrm{~min}$ before adding IFN- $\gamma$. a At day 7 , macrophages were detached and stained with anti-CD 14 PerCP/Cy5.5, antiCD16 FITC, anti-CD86 PerCP/Cy5.5, anti-CD206 FITC, anti-CD163 PE, or anti-HLA-DR FITC and analyzed by using a FACScan flow cytometer (BD Immunocytometry Systems, San Jose, CA, USA). The results are shown as the mean fluorescence intensity (MFI) normalized to the MFI of the isotype control, $n=10$. $\mathbf{b}$ Histograms of a representative experiment are shown. c M1 macrophages differentiated in the presence or absence of ibrutinib $(0.3 \mu \mathrm{M})$ as described above were seeded on top of a thick layer of Matrigel in the upper Transwell chamber. The lower chamber was filled with medium with CCL5. Migration was quantified after 3 days. Results are shown as the percentage of migrated macrophages and representative images are shown in the right panel. Representatives images from the top and inside of matrigel are shown. $\mathbf{d} \mathrm{M} 1$ macrophages were obtained as described above. M2 macrophages were obtained by culturing monocytes from healthy donors with M-CSF $(50 \mathrm{ng} / \mathrm{ml})$ for 7 days and either IL-4 ( $20 \mathrm{ng} / \mathrm{ml})$ or IL-10 $(10 \mathrm{ng} / \mathrm{ml})$ were added during the last 2 days of culture. Ibru-M1 macrophages were treated with ibrutinib during the last 2 days of culture. At day 7, the medium was removed and fresh medium without cytokine was added. Then TNF- $a$ and IL-10 was evaluated after $24 \mathrm{~h}$ of culture by ELISA. Bars show mean \pm SEM. e M1 macrophages differentiated in the presence or absence of ibrutinib $(0.3 \mu \mathrm{M})$ as described above. At day 7 , glucose and lactate concentration in the supernatant was evaluated by using commercial kits. Glucose consumption was calculated as the percentage of glucose in the culture at day 7 relative to the initial glucose concentration in the medium. $\mathbf{f} \mathrm{M} 1$ macrophages were differentiated in the presence or absence of ibrutinib as described before and stimulated with irradiated Mtb-FITC ( $\mathrm{MOl}$ equivalent to 5 ) for $2 \mathrm{~h}$, then cells were trypsinized and the percentage of $\mathrm{FITC}^{+}$macrophages was analyzed by flow cytometry. Results are shown as the percentage of FITC ${ }^{+}$macrophages, $n=10 . \mathbf{g}, \mathbf{h}$ Monocytes from healthy donors were differentiated into $\mathrm{M} 1$ macrophages and were infected with the red fluorescent protein (RFP) expressing M. tuberculosis strain at an $\mathrm{MOI}$ of 5 during $2 \mathrm{~h}$ at $37^{\circ} \mathrm{C}$. Thereafter, ibrutinib at $0.3 \mu \mathrm{M}$ or vehicle was added. After $48 \mathrm{~h}$, the glass coverslips were fixed with PFA $4 \%$ and stained with BODIPY 493/503 (Life Technologies). Finally, slides were mounted and visualized with a FluoView FV1000 confocal microscope (Olympus, Tokyo, Japan) equipped with a Plapon ×60/NA1.42 objective and then analyzed with the software ImageJ-Fiji. $\mathbf{g}$ Quantification of the occupied area with RFP-M. tuberculosis (expressed as Raw Integrated Density) per cell in z-stacks from confocal laser scanning microscopic images. Individual cells were defined by BODIPY-stained cellular membranes that allow us to define the regions of interest for quantification. In all, 80-100 cells of random fields per condition were analyzed. $\mathbf{h}$ Representative microphotographs are shown. $\mathbf{i}$, j Purified $\gamma \delta \mathrm{T}$ cells were stimulated with irradiated Mtb (MOI equivalent to 5) in the presence or absence of ibrutinib. After $24 \mathrm{~h}, \mathrm{CD} 69$ expression and IFN- $\gamma$ production were evaluated by flow cytometry and ELISA, respectively. $\mathbf{i}$ Results are shown as the percentage of $\gamma \delta$ T cells expressing CD69. $\mathbf{j}$ IFN- $\gamma$ concentration in the culture supernatant evaluated by ELISA. ${ }^{*} p<0.05$, Wilcoxon matched-pairs signed rank test. ${ }^{*} p<0.05$, Kruskal-Wallis test, followed by Dunn's multiple comparisons test
\end{abstract}

mechanism might be compromised in ibrutinib-treated patients.

Although ibrutinib treatment in CLL is associated with lower rates of infections compared to the standard chemoimmunotherapy (Fludarabine, Cyclophosphamide, Rituximab), major infections, particularly those of the respiratory tract, are still significant, reported in about one-third of ibrutinib-treated patients ${ }^{8-10}$. The risk of infections appears to be highest during the first 6 months of treatment, and a subsequent improvement in patient's cellular immunity has been suggested by the observation that ibrutinib favors a Th1 polarization ${ }^{1}$ and an increase in the $\mathrm{T}$ cell repertory ${ }^{11}$. Also, recovery from a refractory state of monocytes and $\mathrm{T}$ cells, by the downregulation in programmed cell death protein 1 /programmed cell death ligand 1 expression, was suggested in a study with a small cohort $(n=4)$ of ibrutinib-treated patients ${ }^{12}$. Nevertheless, over the past years, opportunistic infections during ibrutinib treatment such as invasive pulmonary aspergillosis, disseminated fusarium infection, and disseminated cryptococcal disease have been reported ${ }^{13}$, and remarkably in 2015, a case of a CLL patient who developed miliary tuberculosis 1 month after initiation of ibrutinib treatment was reported ${ }^{14}$. In most of those cases, patients did not present neutropenia and showed normal levels of $\mathrm{T}$ cells suggesting that other/s component/s of the immune system might be affected in ibrutinib-treated patients. Moreover, an association between ibrutinib treatment and an increased susceptibility to Pneumocystis jirovecii pneumonia (PCP) in CLL was suggested by Ahn et al. who showed a $5 \%$ of incidence of PCP in a cohort of 96 patients treated with ibrutinib as a single agent ${ }^{15}$. In this report, patients who developed PCP did not have a low $\mathrm{CD}^{+}{ }^{+} \mathrm{T}$ cell count, which is considered the primary risk factor for PCP. Interestingly, alveolar macrophages, TLR2 signaling, and TNF- $\alpha$ secretion are involved in an effective immune response against $P$. jirovecii. Given that TLR2 is a receptor also involved in the recognition of motifs present in several fungi, our finding that ibrutinib impairs macrophages' response through TLR2 might explain, at least in part, the increased susceptibility of ibrutinib-treated patients to PCP and also the presence of other opportunistic fungal infections.

Although further studies are needed to confirm the relevance of our observations in vivo and their impact in clinical practice, we consider that these results represent a warning especially in countries with a high incidence of tuberculosis.

\section{Acknowledgements}

The authors would like to thank to Beatriz Loria, María Tejeda, and Graciela Leiva for their technical assistance. This work was supported by grants and fellowships from the Argentinean National Agency of Promotion of Science and Technology (PICT 1074/2013 and PICT-2015-0055), Fundación Florencio Fiorini, Janssen Non-clinical Investigator Initiated Study (IIS), Consejo Nacional 
de Investigaciones Científicas y Técnicas (CONICET), and the bilateral cooperation programs ECOS-Sud (A14S01).

\section{Author details}

${ }^{1}$ Laboratorio de Inmunología Oncológica, Instituto de Medicina Experimental (IMEX)-CONICET-Academia Nacional de Medicina (ANM), CABA, Argentina.

${ }^{2}$ Laboratorio de Inmunología de Enfermedades Respiratorias, IMEX-CONICETANM, CABA, Argentina. ${ }^{3}$ International Associated Laboratory (LIA) CNRS/ CONICET "IM-TB/HIV" (1167), Toulouse, France. ${ }^{4}$ International Associated Laboratory (LIA) CNRS/CONICET "IM-TB/HIV" (1167), Buenos Aires, Argentina. ${ }^{5}$ Institut de Pharmacologie et de Biologie Structurale, IPBS, Université de Toulouse, CNRS, UPS, Toulouse, France. ${ }^{6}$ Departamento de Fisiología, Biología Molecular y Celular, Facultad de Ciencias Exactas y Naturales, Universidad de Buenos Aires, CABA, Argentina. ${ }^{7}$ IMEX-CONICET-ANM, CABA, Argentina. ${ }^{8}$ Sección de Hematología, Hospital General de Agudos Dr. Teodoro Álvarez, CABA, Argentina. 'Departamento de Hematología, Sanatorio Julio Méndez, CABA, Argentina. ${ }^{10}$ Laboratorio de Inmunidad Innata, MEX-CONICET-ANM CABA, Argentina. ${ }^{11}$ Departamento de Microbiología, Parasitología e Inmunología, Facultad de Medicina, Universidad de Buenos Aires, CABA, Argentina

\section{Conflict of interest}

R.G. receives a Non-clinical Investigator Initiated Study (IIS) grant from Janssen; F.R.B. and R.G. receive compensation as speakers from Janssen. R.G. receives compensation as speaker and as a consultant from Roche and Bristol Myers Squibb. F.R.B. receives compensation as member of Advisory Board from Abbvie. The other authors declare that they have no conflict of interest.

\section{Publisher's note}

Springer Nature remains neutral with regard to jurisdictional claims in published maps and institutional affiliations.

Supplementary Information accompanies this paper at (https://doi.org/ 10.1038/s41408-018-0136-x).

Received: 20 April 2018 Revised: 21 September 2018 Accepted: 9 October 2018

Published online: 05 November 2018

\section{References}

1. Dubovsky, J. A. et al. Ibrutinib is an irreversible molecular inhibitor of ITK driving a Th1-selective pressure in T lymphocytes. Blood 122, 2539-2549 (2013).

2. Fiorcari, S. et al. Ibrutinib modifies the function of monocyte/macrophage population in chronic lymphocytic leukemia. Oncotarget 7, 65968-65981 (2016).

3. Da Roit, F. et al. Ibrutinib interferes with the cell-mediated anti-tumor activities of therapeutic CD20 antibodies: implications for combination therapy. Haematologica 100, 77-86 (2015).

4. Chen, Z. W. Immune regulation of gammadelta T cell responses in mycobacterial infections. Clin. Immunol. 116, 202-207 (2005).

5. Verreck, F. A. et al. Human IL-23-producing type 1 macrophages promote but IL-10-producing type 2 macrophages subvert immunity to (myco)bacteria. Proc. Natl Acad. Sci. USA 101, 4560-4565 (2004).

6. Cougoule, C. et al. Blood leukocytes and macrophages of various phenotypes have distinct abilities to form podosomes and to migrate in 3D environments. Eur. J. Cell Biol. 91, 938-949 (2012).

7. Tan, Z. et al. Pyruvate dehydrogenase kinase 1 participates in macrophage polarization via regulating glucose metabolism. J. Immunol. 194, 6082-6089 (2015).

8. Sun, C. et al. Partial reconstitution of humoral immunity and fewer infections in patients with chronic lymphocytic leukemia treated with ibrutinib. Blood 126, 2213-2219 (2015).

9. Williams, A. M. et al. Analysis of the risk of infection in patients with chronic lymphocytic leukemia in the era of novel therapies. Leuk. Lymphoma $\mathbf{5 9}$ 625-632 (2018)

10. Itchaki, G. \& Brown, J. R. Experience with ibrutinib for first-line use in patients with chronic lymphocytic leukemia. Ther. Adv. Hematol. 9, 3-19 (2018).

11. Yin, Q. et al. Ibrutinib therapy increases $T$ cell repertoire diversity in patients with chronic lymphocytic leukemia. J. Immunol. 198, 1740-1747 (2017).

12. Cubillos-Zapata, C. et al. Ibrutinib as an antitumor immunomodulator in patients with refractory chronic lymphocytic leukemia. Oncoimmunology $\mathbf{5}$, e1242544 (2016).

13. Chamilos, G., Lionakis, M. S. \& Kontoyiannis, D. P. Call for action: invasive fungal infections associated with ibrutinib and other small molecule kinase inhibitors targeting immune signaling pathways. Clin. Infect. Dis. 66, 140-148 (2018).

14. Wang, S. Y. et al. Miliary tuberculosis after initiation of ibrutinib in chronic lymphocytic leukemia. Ann. Hematol. 94, 1419-1420 (2015).

15. Ahn, I. E. et al. Atypical Pneumocystis jirovecii pneumonia in previously untreated patients with CLL on single-agent ibrutinib. Blood 128, 1940-1943 (2016). 